Case Report

\title{
Superior Mesenteric Artery Thrombosis; Rare complication after Pancreaticoduodenectemy
}

\author{
Satyaprakash Jindal', Adithya G.K. ${ }^{2}$, Vachan Hukkeri', Varun Madaan ${ }^{4}$, Chitra $^{5}$, Sandeep Vohra ${ }^{6}$, \\ VivekTandon', Deepak Govil ${ }^{7}$ \\ ${ }^{1,2,3,4}$ DNB trainee, ${ }^{7,8}$ Senior consultant, Department of GI surgery, ${ }^{5}$ DNB trainee, ${ }^{6}$ Senior consultant, Dept of radiology, \\ Indraprastha Apollo Hospital, Delhi.
}

Corresponding Author : Adithya G.K., DNB Trainee, Department of Gl surgery, Indraprastha Apollo Hospital, Delhi.

E-mail: adi3anny@gmail.com

Received

$: 10.10 .2017$

Review Completed : 12.12.2017

Accepted

: 28.01.2018

Keywords : SMA thrombosis, Pancreaticoduodenectemy, cholangiocarcinoma,

\begin{tabular}{|c|}
\hline Access this article online \\
\hline Quick Response Code \\
\hline
\end{tabular}

\section{Introduction}

Pancreaticoduodenectemy(PD) is the treatment of choice for malignantlesions of the pancreas and periampullary region. PD consists of multi visceral dissection and associated with high morbidity. Common complications associated with this morbid procedure include delayed gastric emptying, pancreatic fistula, anastomotic leak and intra abdominal collection. Other medical complications include arrhythmia, renal failure, pulmonary complications, UTI and Deep vein thrombosis. Here we present a case of Superior mesenteric artery (SMA) thrombosis after PD done for Lower end cholangiocarcinoma, which has ever been reported in literature.

\section{Case Report}

A 68 years old female presented with obstructive jaundice, anorexia and weight loss for 2 months. She was diagnosed with lower end cholangiocarcinoma. She had no other comorbidity with ECOG1. Her serum bilirubin was $30 \mathrm{mg} / \mathrm{dl}$ and CA19.9 level was 418.5. Preoperative imaging was consistent with cholangiocarcinoma without any evidence of vascular pathology. Preoperative biliary drainage was done and patient was optimized for surgery. She underwent classic PD with feeding jejunostomy. Intraoperatively, the lesion was well localized to periampullary region and there was no evidence of any vascular involvement. Patient tolerated the procedure well without any intraoperative complication.

Thromboprophylaxis was given in perioperative period with LMWH and pneumatic pump. She was started on enteral feeding by feeding jejunostomy on $4^{\text {th }}$ POD, which she tolerated well. Her initial postoperative course was smooth, but she developed drowsiness on POD-8. Beside that she was haemo dynamically stable and her biochemical parameters were normal. In view of persistent drowsiness, neurological evaluation was done including CT 
scan of head, which revealed no abnormality. On next day she became progressively unresponsive and developed abdominal distension. She had gastrointestinal bleed, which precipitate as malena and hemorrhagic aspirate from Nasogastric tube. CT abdomen was done which revealed SM A thrombosis without any evidence of bowel gangrene. In view of poor general condition, she was managed conservatively with anti-coagulative therapy. But patient progressively deteriorated and succumb to death within 24 hrs. We retrospectively evaluated for any predisposing factor for vascular thrombosis, but were unable to find any obvious cause for SM A thrombosis in this patient.

\section{Discussion}

Pancreaticoduodenectemy (PD) is recommended treatment for malignant diseases of periampullary region. In the past decades postoperative mortality rates upto $40 \%$ have been reported for this morbid procedure, but afterimprovement of technical and perioperative care, thishas been reduced toless than $5 \%$ in high-volume centers. (1)Despite these advances, morbidity still remains high, with reported complication rate of $30 \%$ to $60 \%$.The majority of perioperative complications after PD are not life threatening, though they result in prolonged hospital stay and readmissions. (2)The most common complication reported is delayed gastric emptying, seen in 30-35\% cases. Other associated surgical complications are anastomotic failure, among which pancreatico enteric anastomotic leak is most common followed by gastro-jejunostomy and choledoco/hepaticojejunostomy. Other nonsurgical complications in these patients consisted of cardiac complications like cardiac arrhythmias, pneumonia, hemorrhageand renal failure. Other reported complications after PD are pulmonary embolism, myocardial infarction and splanchnic vein thrombosis. (3)

Portal vein and superior mesenteric vein thrombosis has been reported after PD in literature. As increased experience with vascular resection and reconstruction during PD for borderline resectable tumors, there is an increasing trend toward these complications. SMA thrombosis as a complication of $\mathrm{PD}$, is rarely been reported in literature. Although there is theoretical risk of SMA thrombosis after SM A reconstruction, but there is paucity of data on this complication. Arterial thrombosis can be precipitated due to postoperative hypovolemia in patients with pre-existing atherosclerosis.Our patient underwent classical PD without any vascular intervention orintraoperative complication. Preoperatively patient was otherwise fit without any comorbidity and initial postoperative period was smooth. There was no evidence of any vascular disease including atherosclerosis in this patient.

SM A thrombosis is characterized by a high mortalityrate. Although relatively less commonly seen in clinical practice, SMA thrombosis is the most common cause of acute mesenteric ischemia.Thrombosisin SM A most commonly occurs at the level ofostia and results in ischemia of the entire midgut, which consequently leads to infarction if left untreated. Prognosis of acute thrombotic ischemia is worse even after restoration of mesenteric blood supply (4).

Patients with SMA thrombosis share the common features associated with atherosclerotic disease such as hypertension, hyperlipidemia, diabetes and smoking. These patients often have a past history of other vascular event like myocardial infarction; stroke or peripheral vascular disease (5).Acute SMA thrombosis commonly occurs in a patient ofchronic mesenteric ischemia and frequently precipitated by intravascular volume depletion from dehydration. This dehydration can occur after severe diarrhea or vomiting associated with gastrointestinal diseases, postoperative fluid lossesand sepsis or due to third space fluid loss, such as acute pancreatitis.Clinical symptoms of SM A thrombosis are nonspecific, which can leads to delay in diagnosis. Patients often present with severe abdominal pain that is out of proportional to clinical finding. (6)

A high index of suspicion is required to diagnosis of SMA thrombosis.Angiography is considered as the "gold standard" for diagnosing mesenteric vascular occlusion. 
But now a day's MDCT Angiographyhas become investigation of choice with high specificity. In a recent meta-analysis, MDCT Angiography had a sensitivity and specificity of $93.3 \%$ and $95.9 \%$ respectively (7). An aggressive approach to resuscitation and hemodynamic monitoring is strongly encouraged in these patients.Intraarterial administration of thrombolytic agents has effectively restored mesenteric blood flow in selected patients when administered within hours of symptom onset. The goals of surgical therapy are to restore mesenteric blood flow, assess intestinal viability, and resect nonviable or necrotic intestine. Endarterectomy or ante grade bypass using a prosthetic conduit are the revascularization procedures of choice in the absence of enteric contamination. Endovascular treatments, such as thrombolysis, are generally avoided owing to the urgent need to restore intestinal perfusion and prevent intestinal infarction (8).

\section{References}

1. Van Heek NT, Kuhlmann KF, Scholten RJ, de Castro SM, Busch OR, van Gulik TM, et al.Hospital volume and mortality after pancreatic resection: a systematic review and an evaluation of intervention in the Netherlands. Ann Surg. 2005;242(6):781-8.

2. Lin JW, Cameron JL, Yeo CJ, et al. Risk factors and outcomes in post pancreaticoduodenectomy pancreaticocutaneous fistula. J Gastrointest Surg 2004;8(8):951-9.

3. Saraee et al. Whipple procedure: a review of a 7 -year clinical experience in a referral center for hepatobiliary and pancreas diseases World Journal of Surgical Oncology (2015) 13:98 DOI 10.1186/s12957015-0523-8

4. Schoots IG, Koffeman GI, Legemate DA, Levy M, Van Gulik TM: Systematic review of survival after acute mesenteric ischemia according to disease aetiology. Br J Surg 2004, 91:17-21.
SMA thrombosis is associated with high mortality and earlier studies reported mortality rate up to $80-90 \%$. Conservative treatment results in a mortality of $100 \%$ with rare exceptions. In patients treated with bowel resection, short term survival can be achieved in $49 \%$ of the cases. Despite advances in surgical and diagnostic management, literature reported a short-term mortality rate of $30-60 \%$ in patients undergoing revascularization of the SM A (9).

\section{Conclusion}

Pancreaticoduodenectemy is a morbid procedure and associated with some known complications. However, SM A thrombosis is a rare complication after PD. These patients often have unusual presentation, so investigations like CT abdomen are recommended earlier in case of any suspicion. SMA thrombosis is associated with high mortality and these patients are difficult to salvage.

5. Jarvinen0, Laurikka J, Salenius JP, Tarkka M.Acute intestinal ischaemia.A review of 214 cases. Ann ChirGynaecol. 1994;83(1):22-5.

6. Charles J. Shanley, MD, FACS*, Jeffrey B. Weinberger, MD Acute Abdominal Vascular Emergencies M ed Clin N Am 92 (2008) 627-647

7. MenkeJ. Diagnostic accuracy of multidetector CT in acute mesenteric ischemia: systematic review and meta-analysis. Radiology. 2010; 256(1):93-101.

8. Schoots IG, Levi M M, Reekers JA, et al. Thrombolytic therapy for acute superior mesentericartery occlusion. J VascIntervRadiol 2005;16(3):317-29.

9. Bjorck M, Acosta S, Lindberg F, Troeng T, Bergqvist D. Revascularization of the superior mesenteric artery after acute thromboembolic occlusion. 